\title{
Separable Brane Cosmologies in Heterotic M-Theory
}

\author{
James E. Lidsey ${ }^{1}$ \\ Astronomy Unit, School of Mathematical Sciences, \\ Queen Mary 83 Westfield, Mile End Road, LONDON, E1 4NS, U.K.
}

\begin{abstract}
It is shown that any anisotropic and inhomogeneous cosmological solution to the lowest-order, four-dimensional, dilaton-graviton string equations of motion may be employed as a seed to derive a curved, three-brane cosmological solution to five-dimensional heterotic M-theory compactified on a Calabi-Yau threefold. This correspondence formally relates a weakly coupled string cosmology directly with a strongly coupled one. The asymptotic behaviour of a wide class of spatially homogeneous braneworlds is deduced. Similar solutions may be derived in toroidally compactified massive type IIA supergravity.
\end{abstract}

PACS numbers: $98.80 . \mathrm{Cq}, 11.25 . \mathrm{Mj}, 04.50 .+\mathrm{h}$

${ }^{1}$ Electronic mail: jel@maths.qmw.ac.uk 
The $\mathrm{E}_{8} \times \mathrm{E}_{8}$ heterotic string theory is phenomenologically favoured from a particle physics perspective, because it may contain the standard model of gauge interactions [1]. The strongly coupled regime of this theory has been interpreted by Hořava and Witten as M-theory on the orbifold $\mathrm{R}^{10} \times S^{1} / \mathrm{Z}_{2}$ with an infra-red limit corresponding to eleven-dimensional supergravity on a manifold with two ten-dimensional boundaries [2]. A set of $\mathrm{E}_{8}$ gauge supermultiplets on each of the orbifold fixed planes ensures the cancellation of anomalies. Compactification of this theory on a Calabi-Yau three-fold results in a gauged, five-dimensional supergravity theory with two fourdimensional boundaries [3, 4]. The theory does not admit five-dimensional Minkowski space as a consistent solution. Instead the 'vacuum' corresponds to a static BPS state given by a warped product of four-dimensional flat space and an interval [四. Such a solution may be interpreted as a pair of parallel three-branes with equal and opposite tension that are located at the orbifold planes. Our observed four-dimensional spacetime is then interpreted as the world--volume of one of these branes.

This interpretation has significant implications for early universe cosmology and it is therefore important to derive cosmological models in this context. Such a study involves a search for time-dependent solutions to the field equations. Cosmological solutions in heterotic M-theory have been found previously by reinterpreting temporal and radial coordinates in certain $p$-brane backgrounds [5]. An alternative method was adopted by Lukas et al., who found spatially flat Friedmann-Robertson-Walker (FRW) cosmological brane solutions by compactifying Hořava-Witten theory on a Calabi-Yau space [6]. This approach was subsequently generalized to the spatially curved, FRW models by Reall [7]. Domain wall solutions moving in a time-dependent bulk have also been found [8] and a solution preserving $N=1$ supersymmetry in four dimensions was recently presented [9].

It is reasonable to suppose that spatial anisotropies and inhomogeneities in our three-dimensional subspace would have been significant in the environment of the very early universe and it is therefore important to develop models that take these effects into account. In view of this, we derive a wide class of spatially anisotropic and inhomogeneous brane cosmologies in Hořava-Witten M-theory. We consider the class of five-dimensional models with an action of the form

$$
S=\frac{1}{2} \int d^{4} x \int_{-\pi}^{+\pi} d y \sqrt{-g}\left[R-\frac{1}{2}(\nabla \varphi)^{2}-\frac{\alpha^{2}}{3} e^{-a \varphi}\right]+\sum_{i=1}^{2} \int_{\mathcal{M}_{4}^{(i)}} d^{4} x \sqrt{-g_{i}} \mu_{i} e^{-a \varphi / 2}
$$

where $R$ is the Ricci scalar of the bulk spacetime with metric $g_{A B}, g \equiv \operatorname{det} g_{A B}$ and $\left\{a, \mu_{i}\right\}$ are constants . The orbifold, $S^{1} / \mathrm{Z}_{2}$, is parametrized by the coordinate, $y$, and corresponds to a segment of the real line bounded by two fixed points on the circle. It is defined over the coordinate range $y \in[-\pi, \pi]$, where the endpoints are identified

\footnotetext{
${ }^{1}$ In this paper, the spacetime metric has signature $(-,+,+, \ldots,+)$. Upper case, Latin indices without a circumflex accent vary from $A=(0,1, \ldots, 4)$, upper case Latin indices with circumflex accents take values in the range $\hat{A}=(0,1, \ldots, 10)$, lower case Greek indices span $\mu=(0,1,2,3)$ and lower case Latin indices denote spatial dimensions.
} 
and the discrete symmetry $y \rightarrow-y$ is imposed. This transformation reverses the orientation of the circle and the fixed points are specified by the conditions $y=\{0, \pi\}$. These coordinates determine the location of the four-dimensional hyperplanes, $\mathcal{M}_{4}^{(i)}$. The metrics on these orbifold fixed planes are defined by $g_{\mu \nu}^{(1)} \equiv g_{\mu \nu}(y=0)$ and $g_{\mu \nu}^{(2)} \equiv g_{\mu \nu}(y=\pi)$, respectively, and $g^{(i)} \equiv \operatorname{det} g_{\mu \nu}^{(i)}$. For consistency, we further require that all degrees of freedom are invariant under the $\mathrm{Z}_{2}$ transformation.

When $a=2$ and $\mu_{1}=-\mu_{2}=\sqrt{2} \alpha$, action (11) represents a truncation of Hořava-Witten theory compactified on a Calabi-Yau three-fold [4], where the elevendimensional metric is given by

$$
d \hat{s}_{11}^{2}=e^{-2 \varphi / 3} g_{A B} d x^{A} d x^{B}+e^{\varphi / 3} \Omega_{m n} d z^{m} d z^{n}
$$

and the Calabi-Yau space has a metric $\Omega_{m n}=\Omega_{m n}\left(z^{p}\right)$ [4]. The radius of the CalabiYau space is parametrized by the scalar field, $\varphi$, that self-interacts via a Liouville potential. This interaction potential arises because a non-zero internal component of the four-form field strength must be included if the compactification from eleven to five dimensions is to be consistent [4]. This contribution is parametrized by the constant, $\alpha$, that specifies the brane tensions.

In deriving the field equations from the action (1), we assume that the constants $\left\{a, \mu_{i}\right\}$ are arbitrary. The gravitational and moduli field equations then take the form

$$
\begin{gathered}
G_{A B}=\frac{1}{2} \nabla_{A} \varphi \nabla_{B} \varphi-g_{A B}\left(\frac{1}{4}(\nabla \varphi)^{2}+\frac{\alpha^{2}}{6} e^{-a \varphi}\right) \\
+g_{\mu A} g_{\nu B} e^{-a \varphi / 2}\left[\mu_{1} \delta(y) g_{(1)}^{\mu \nu} \sqrt{g_{1} / g}+\mu_{2} \delta(y-\pi) g_{(2)}^{\mu \nu} \sqrt{g_{2} / g}\right] \\
\nabla^{2} \varphi=-\frac{a \alpha^{2}}{3} e^{-a \varphi}+a e^{-a \varphi / 2}\left[\mu_{1} \delta(y) \sqrt{g_{1} / g}+\mu_{2} \delta(y-\pi) \sqrt{g_{2} / g}\right],
\end{gathered}
$$

respectively.

We search for cosmological solutions where the five-dimensional metric has the general form

$$
d s_{5}^{2}=H^{4 /(3 \Delta)} f_{\mu \nu} d x^{\mu} d x^{\nu}+H^{16 /(3 \Delta)} e^{2 \beta} d y^{2}
$$

and the constant

$$
\Delta \equiv \frac{3 a^{2}-8}{3}
$$

is assumed to be non-zero. The warp factor, $H=H(y)$, depends only on the orbifold coordinate and the four-dimensional metric, $f_{\mu \nu}=f_{\mu \nu}\left(x^{\lambda}\right)$, and scalar function, $\beta=$ $\beta\left(x^{\mu}\right)$, are independent of this variable. The components of the five-dimensional Einstein tensor for the metric (5) are

$$
G_{\mu \nu}=\bar{G}_{\mu \nu}-\bar{\nabla}_{\mu \nu} \beta-\bar{\nabla}_{\mu} \beta \bar{\nabla}_{\nu} \beta+f_{\mu \nu}\left[\bar{\nabla}^{2} \beta+(\bar{\nabla} \beta)^{2}\right]
$$




$$
\begin{array}{r}
+\frac{2}{\Delta}\left[\frac{H^{\prime \prime}}{H}-\left(1+\frac{4}{3 \Delta}\right) \frac{H^{2}}{H^{2}}\right] e^{-2 \beta} H^{-4 / \Delta} f_{\mu \nu} \\
G_{\mu y}=\frac{2}{\Delta} \frac{H^{\prime}}{H} \bar{\nabla}_{\mu} \beta \\
G_{y y}=-\frac{1}{2} \bar{R} H^{4 / \Delta} e^{2 \beta}+\frac{8}{3 \Delta^{2}} \frac{H^{\prime 2}}{H^{2}}
\end{array}
$$

where a bar indicates that quantities are constructed from the world--volume metric, $f_{\mu \nu}$, and a prime denotes differentiation with respect to the orbifold coordinate, $y$.

In this work, we assume a separable ansatz for the Calabi-Yau modulus field: $\varphi \equiv \varphi_{1}(x)+\varphi_{2}(y)$. As shown recently by Brecher and Perry [10], there exists a class of three-brane (domain wall) solutions to Eqs. (3) and (14) for the Hořava-Witten theory when $f_{\mu \nu}$ is a Ricci-flat metric, $\beta=\varphi_{1}=0, \varphi_{2}=3 \ln H$ and $H=1+(\sqrt{2} \alpha|y|) / 3$. The metric, $f_{\mu \nu}$, may then be interpreted as the world-volume of the branes. When the world-volume is Minkowski space, the solution reduces to the BPS state of Ref. [4]. We generalize this class of solution such that the Calabi-Yau radius and orbifold dimension have a non-trivial dependence on the world-volume coordinates.

The $(\mu y)$-component of the Einstein field equations (3) then reduces to

$$
\frac{H^{\prime}}{H} \bar{\nabla}_{\mu} \beta=\frac{\Delta}{4} \varphi_{2}^{\prime} \bar{\nabla}_{\mu} \varphi_{1}
$$

and Eq. (10) is solved by

$$
\begin{array}{r}
\varphi_{2}=\frac{2 a}{\Delta} \ln H \\
\varphi_{1}=\frac{2}{a} \beta
\end{array}
$$

for $a \neq 0$. By substituting Eqs. (7), (9), (11) and (12) into the Einstein equations (3), it follows that the remaining non-trivial components then take the form

$$
\begin{gathered}
\bar{G}_{\mu \nu}-\left(1+\frac{2}{a^{2}}\right) \bar{\nabla}_{\mu} \beta \bar{\nabla}_{\nu} \beta-\bar{\nabla}_{\mu \nu} \beta+f_{\mu \nu}\left[\bar{\nabla}^{2} \beta+\left(1+\frac{1}{a^{2}}\right)(\bar{\nabla} \beta)^{2}\right] \\
=\left[-\frac{2}{\Delta} \frac{H^{\prime \prime}}{H}+\frac{1}{H}\left(\mu_{1} \delta(y)+\mu_{2} \delta(y-\pi)\right)+\frac{1}{\Delta} \frac{H^{\prime 2}}{H^{2}}-\frac{\alpha^{2}}{6 H^{2}}\right] e^{-2 \beta} H^{-4 / \Delta} f_{\mu \nu} \\
\frac{1}{2} \bar{R}-\frac{1}{a^{2}}(\bar{\nabla} \beta)^{2}=-\left[\frac{1}{\Delta} \frac{H^{\prime 2}}{H^{2}}-\frac{\alpha^{2}}{6 H^{2}}\right] e^{-2 \beta} H^{-4 / \Delta},
\end{gathered}
$$

where we have employed Eq. (6). Moreover, the field equation (国) for the Calabi-Yau radius simplifies to

$$
\begin{array}{r}
\bar{\nabla}^{2} \beta+(\bar{\nabla} \beta)^{2}=-\frac{a^{2}}{2} e^{-\beta} H^{-4 / \Delta}\left[\frac{2}{\Delta} \frac{H^{\prime \prime}}{H}-\frac{1}{H}\left(\mu_{1} \delta(y)+\mu_{2} \delta(y-\pi)\right)\right. \\
\left.-\frac{2}{\Delta} \frac{H^{\prime 2}}{H^{2}}+\frac{\alpha^{2}}{3 H^{2}}\right] e^{-2 \beta} H^{-4 / \Delta} .
\end{array}
$$


The left- and right-hand sides of each of Eqs. (13)-(15) can be separated by equating them all to zero. This separates terms that depend only on the orbifold coordinate from those that are independent of $y$. The right-hand sides vanish if

$$
\begin{array}{r}
H^{\prime 2}=\frac{\Delta \alpha^{2}}{6} \\
H^{\prime \prime}=\frac{\Delta}{2}\left(\mu_{1} \delta(y)+\mu_{2} \delta(y-\pi)\right)
\end{array}
$$

are simultaneously satisfied and the left-hand sides are solved by

$$
\begin{aligned}
& \bar{R}_{\mu \nu}=\bar{\nabla}_{\mu \nu} \beta+\left(1+\frac{2}{a^{2}}\right) \bar{\nabla}_{\mu} \beta \bar{\nabla}_{\nu} \beta \\
& \bar{\nabla}^{2} \beta+(\bar{\nabla} \beta)^{2}=0 .
\end{aligned}
$$

The solution to Eq. (16) satisfying the orbifold symmetry is [4, 11

$$
H=1+m|y|, \quad m \equiv \sqrt{\frac{\alpha^{2} \Delta}{6}} .
$$

Differentiating Eq. (201) twice with respect to the orbifold coordinate results in two $\delta$ functions [2, $^{2} \delta(y)$ and $\delta(y-\pi)$. Consistency with Eq. (17) then implies that the brane tensions must satisfy $\mu_{1}=-\mu_{2}=\left(8 \alpha^{2} / 3 \Delta\right)^{1 / 2}$. When $a=2$, these are precisely the conditions arising in the compactified Hořava-Witten heterotic theory [4]. The functional form of the $y$-dependent part of the Calabi-Yau modulus field is then directly deduced from Eq. (IT). Thus, the orbifold-dependent sector of the solution represents a pair of parallel three-branes and has the functional form of the vacuum solution of Ref. [4].

The dynamics of the branes is completely determined by the solutions to Eqs. (18)-(19) and it only remains to solve this system of equations. When the length of the orbifold interval is independent of the world-volume coordinates, Eq. (18) implies that $\bar{R}_{\mu \nu}=0$ and we recover the class of Ricci-flat branes found by Brecher and Perry [10]. Further insight may be gained by performing the conformal transformation

$$
{ }^{(s)} f_{\mu \nu}=\Omega^{2} f_{\mu \nu}, \quad \Omega^{2} \equiv e^{b \Phi}
$$

on the world-volume metric, where

$$
\Phi \equiv \frac{\beta}{b-1}, \quad b \equiv 1+\frac{a}{\sqrt{4+3 a^{2}}} .
$$

\footnotetext{
${ }^{2}$ We employ the expressions $|y|^{\prime}=\epsilon(y)-\epsilon(y-\pi)-1$ and $|y|^{\prime \prime}=2 \delta(y)-2 \delta(y-\pi)$, where $\epsilon(y)=1$ if $y \geq 0$ and $\epsilon(y)=-1$ if $y<0$. The second $\delta$-function arises because $y$ is periodic.
} 
Substituting in the separable condition (12) implies that Eqs. (18)-(19) then reduce to

$$
\begin{aligned}
& { }^{(s)} R_{\mu \nu}=-{ }^{(s)} \nabla_{\mu \nu} \Phi \\
& { }^{(s)} \nabla^{2} \Phi=\left({ }^{(s)} \nabla \Phi\right)^{2}
\end{aligned}
$$

Eqs. (23) and (24) represent the lowest-order $\beta$-function equations for the massless graviton $\left({ }^{(s)} f_{\mu \nu}\right)$ and dilaton $(\Phi)$ excitations of the heterotic string compactified on a static Calabi-Yau manifold in the absence of the Neveu-Schwarz/Neveu-Schwarz two-form potential. They are valid in the perturbative, weakly coupled regime of the theory. Given a solution to these equations, we may immediately deduce the functional form of the five-dimensional brane metric (5) and the solution may be reinterpreted in an eleven-dimensional context by substituting Eq. (5) into Eq. (2). We find for the Hořava-Witten case that

$$
d \hat{s}_{11}^{2}=H^{-1} e^{-11 \Phi / 6}\left[{ }^{(s)} f_{\mu \nu} d x^{\mu} d x^{\nu}\right]+H^{2} e^{2 \Phi / 3} d y^{2}+H e^{\Phi / 6} \Omega_{m n} d z^{m} d z^{n},
$$

where the warp factor is given by Eq. (20).

Thus, it follows that any solution to the lowest-order, dilaton-graviton $\beta$-function equations may be related, after appropriate field redefinitions, to a curved, threebrane background of heterotic $M$-theory compactified on a Calabi-Yau three-fold. The solution (25) provides a direct relationship between a given heterotic string cosmology that is valid in the weakly coupled regime of the theory and a brane cosmology that is valid in the strongly coupled limit. The physical separation between the two branes becomes larger as the string coupling increases and varies as $r \propto g_{s}^{2 / 3}$, where $g_{s}^{2} \equiv e^{\Phi}$ parametrizes the string coupling in the weakly coupled solution. The separable condition (12) implies that the radius of the Calabi-Yau space must follow the behaviour of this coupling in a well defined way. For the specific case of the spatially flat, FRW model, the weakly coupled solution to Eqs. (23) and (24) is given by the 'dilaton-vacuum' solution, $a_{s} \propto \eta^{(1 \pm \sqrt{3}) / 2}$ and $e^{\Phi} \propto \eta^{ \pm \sqrt{3}}$, where $a_{s}$ represents the string-frame scale factor and $\eta$ is conformal time. This solution forms the basis of the pre-big bang inflationary scenario [12] and we recover the brane cosmology of Ref. [6] when this solution is transformed into Eq. (25).

A further conformal transformation given by

$$
\tilde{f}_{\mu \nu}=\Theta^{2(s)} f_{\mu \nu}, \quad \Theta^{2} \equiv e^{-\Phi}
$$

implies that Eqs. (23) and (24) are dynamically equivalent to

$$
\begin{array}{r}
\tilde{R}_{\mu \nu}=\frac{1}{2} \tilde{\nabla}_{\mu} \Phi \tilde{\nabla}_{\nu} \Phi \\
\tilde{\nabla}^{2} \Phi=0 .
\end{array}
$$

Eqs. (27)-(28) represent the field equations for a massless scalar field, $\Phi$, that is minimally coupled to Einstein gravity. This correspondence is important because the 
four-dimensional, Einstein-massless scalar field system has been extensively studied in the literature, both within the anisotropic and inhomogeneous cosmological settings [13. This implies that known techniques for generating solutions and analyzing the asymptotic behaviour and singular nature of cosmological models in standard general relativity may be applied directly to the class of brane cosmologies derived above.

For example, in the case of the spatially homogeneous Bianchi universes, the massless scalar field may be interpreted as a stiff perfect fluid, where the speed of sound in the fluid is equal to the speed of light. A number of exact solutions are known for this effective equation of state [13]. Moreover, the generic asymptotic behaviour of many of these cosmologies has been established. In particular, apart from a set of measure zero, all orthogonal Bianchi class B cosmologies (types IV, V, $\mathrm{VI}_{h}$ and $\mathrm{VII}_{h}$ ) containing a stiff perfect fluid, where the fluid velocity is orthogonal to the surfaces of homogeneity, are asymptotic in the future to a plane wave state and asymptotic in the past to the Jacobs Bianchi type I solution [14].

The four-dimensional Jacobs type I metric [15] is the generalization of the vacuum Kasner [16] solution to include a stiff perfect fluid. In a string cosmological context, it is conformally equivalent to the 'rolling radii' solution found by Meuller [17]. The string-frame metric has the form ${ }^{(s)} d s_{4}^{2}=-d t_{s}^{2}+\sum_{i=1}^{3} t_{s}^{2 p_{i}} d x_{i}^{2}$ and the dilaton field is given by $e^{-\Phi} \propto t_{s}^{p}$, where $\sum_{i=1}^{3} p_{i}^{2}=1$ and $\sum_{i=1}^{3} p_{i}=1-p$. In general, there is a curvature singularity in the four-dimensional metric at $t_{s}=0$. Since the fivedimensional Ricci scalar is given by $R=H^{-1} \bar{R}+\ldots$, the solution also admits a five-dimensional singularity. Indeed, a similar conclusion holds in eleven dimensions because, in general, the square of the Riemann tensor varies as $\hat{R}_{\hat{A} \hat{B} \hat{C} \hat{D}^{\hat{R}}} \hat{A}^{\hat{A} \hat{C} \hat{D}} \propto t_{s}^{-4}$. Indeed, in the absence of any dependence on the orbifold coordinate, the line-element (25) in the early-time limit of these models would correspond to a specific Kasner solution of eleven-dimensional, vacuum Einstein gravity.

In principle, strongly coupled, spatially inhomogeneous brane cosmologies may also be derived in the manner outlined above. The simplest class of inhomogeneous cosmologies that generalizes the Bianchi universes are the diagonal Einstein-Rosen $G_{2}$ models [18, 19]. These admit two commuting spacelike Killing vectors and have a metric of the general form $d s^{2}=e^{2 f(t, x)}\left(-d t^{2}+d x^{2}\right)+t\left(e^{p(t, x)} d y^{2}+e^{-p(t, x)} d z^{2}\right)$, where $\{f, p\}$ are scalar functions. Thus, spatial homogeneity is broken along the $x^{-}$ direction. Numerous techniques exist for generating a minimally coupled, scalar field $G_{2}$ solution from a corresponding vacuum solution of this form. (For a review, see, e.g., Ref. [19]). This class of models is interesting because there exists a long standing conjecture that they represent the leading-order approximation to the general solution of Einstein gravity in the vicinity of the curvature singularity [20]. An investigation into string cosmologies of this type is therefore important and a number of recent studies appropriate to the weakly coupled regime have recently been presented [21]. It would be interesting to perform a detailed investigation into the singular nature of the corresponding strongly coupled models, although this is beyond the scope of the present work. It would also be interesting to further generalize the analysis to 
include cosmologies with only a single isometry. These models could be generated from vacuum $G_{2}$ backgrounds by employing the algorithm recently developed by Lazkoz 22].

Brane cosmologies may also be found in other supergravity theories in the manner outlined in this work. A particular example is Romans' massive type IIA theory in ten dimensions [23]. This theory represents the low-energy limit of the type IIA superstring [24]. After a suitable truncation, the toroidal compactification of this theory to five dimensions results in an effective bulk action given by the five-dimensional part of Eq. (1), where $a^{2}=20 / 3(\Delta=4)$ [25]. Indeed, this value for the coupling parameter arises in all massive supergravity theories derived from the Scherk-Schwarz compactification of a higher-dimensional theory containing an axion field 25]5.

In conclusion, therefore, we have shown that Hořava-Witten theory admits a wide class of anisotropic and inhomogeneous cosmological solutions if the Calabi-Yau radius and orbifold dimension scale in a specific way. We have directly related these strongly coupled backgrounds to weakly coupled, four-dimensional string cosmologies. These models therefore provide a dynamical mechanism for interpolating between the weakly and strongly coupled limits of the $\mathrm{E}_{8} \times \mathrm{E}_{8}$ heterotic string theory.

The author is supported by the Royal Society. We thank D. Clancy, R. Lazkoz and G. Pollifrone for helpful discussions.

\section{References}

1. Green M B, Schwarz J H and Witten E 1987 Superstring Theory (Cambridge: Cambridge University Press) Polchinski J 1998 String Theory (Cambridge: Cambridge University Press)

2. Hořava P and Witten E 1996 Nucl. Phys. B460 506 Hořava P and Witten E 1996 Nucl. Phys. B475 94

3. Witten E 1996 Nucl. Phys. B471 135

4. Lukas A, Ovrut B A, Stelle K S and Waldram D 1999 Phys. Rev. D59 086001

5. Benakli K 1999 Int. J. Mod. Phys. D8 153

Benakli K 1999 Phys. Lett. B447 51

6. Lukas A, Ovrut B A and Waldram D 1999 Phys. Rev. D60 086001

\footnotetext{
${ }^{3}$ In the Scherk-Schwarz compactification [26], the axion is assumed to have a linear dependence on an internal coordinate. This is manifested in the lower-dimensional theory as an exponential self-interaction potential for the modulus field. The reader is referred to Ref. [25] for details.
} 
7. Reall H S 1999 Phys. Rev. D59 103506

8. Chamblin H A and Reall H S 1999 hep-th/9903225

9. Meissner K A and Olechowski M 1999 hep-th/9910161

10. Brecher D and Perry M J 1999 hep-th/9908018

11. Lü H, Pope C N, Sezgin E and Stelle K S 1995 Nucl. Phys. B456 669

12. Veneziano G 1991 Phys. Lett. B265 287

Gasperini M and Veneziano G 1993 Astropart. Phys. 1317

13. Kramer D, Stephani H, MacCallum M and Herlt E 1980 Exact Solutions of Einstein's Equations (Cambridge: Cambridge University Press)

14. Hewitt C G and Wainwright J 1993 Class. Quantum Grav. 1099

15. Jacobs K C 1968 Astrophys. J. 153661

16. Kasner E 1925 Am. Math. Soc. 27155

17. Mueller M 1990 Nucl. Phys. 33737

18. Carmeli M, Charach Ch and Malin S 1981 Phys. Rep. 7679 Verdaguer E 1993 Phys. Rep. 2291

19. Krasinski A 1997 Inhomogeneous Cosmological Models (Cambridge: Cambridge University Press)

20. Belinskii V A and Khalatnikov I M 1973 Sov. Phys. JETP 36591 Belinskii V A, Khalatnikov I M and Lifshitz E M 1982 Adv. Phys. 31639 Berger B K, Garfinkle D, Isenberg J, Moncrief V and Weaver M 1998 Mod. Phys. Lett. A13 1565

21. Barrow J D and Kunze K 1997 Phys. Rev. D56 741

Feinstein A, Lazkoz R and Vazquez-Mozo M A 1997 Phys. Rev. D56 5166

Clancy D, Feinstein A, Lidsey J E and Tavakol R 1999 Phys. Rev. D60 043503

22. Lazkoz R 1999 Phys. Rev. D60 104008

23. Romans L J 1986 Phys. Lett. B169 374

24. Polchinski J 1995 Phys. Rev. Lett. 754724

25. Cowdall P M, Lü H, Pope C N, Stelle K S and Townsend P K 1997 Nucl. Phys. B486 49

26. Scherk J and Schwarz J H 1979 Phys. Lett. B82 60 